\title{
Clinical Features of Male Breast Cancer: Experiences from Seven Institutions Over 20 Years
}

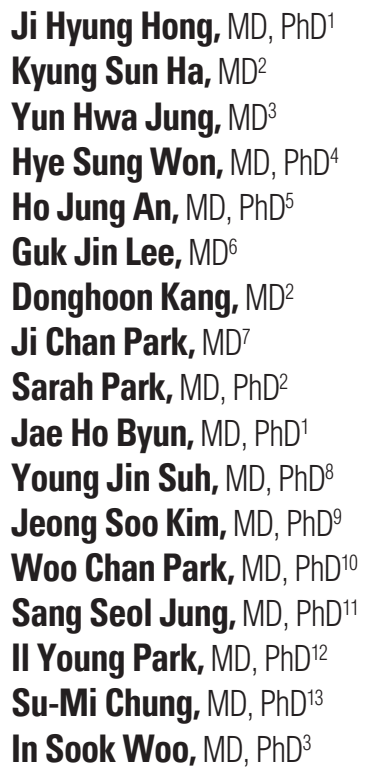

${ }^{*}$ A list author's affiliations appears at the end of the paper.

\section{Purpose}

Breast cancer treatment has progressed significantly over the past 20 years. However, knowledge regarding male breast cancer $(\mathrm{MBC})$ is sparse because of its rarity. This study is an investigation of the clinicopathologic features, treatments, and clinical outcomes of MBC.

\section{Materials and Methods}

Clinical records of 59 MBC patients diagnosed during 1995-2014 from seven institutions in Korea were reviewed retrospectively.

\section{Results}

Over a 20-year period, MBC patients accounted for 0.98\% among total breast cancer patients, and increased every 5 years. The median age of MBC patients was 66 years (range, 24 to 87 years). Forty-three patients (73\%) complained of a palpable breast mass initially. The median symptom duration was 5 months (range, 1 to 36 months). Mastectomy was performed in $96 \%$ of the patients. The most frequent histology was infiltrating ductal carcinoma (75\%). Ninety-one percent of tumors (38/43) were estrogen receptor-positive, and $28 \%(11 / 40)$ showed epidermal growth factor receptor 2 (HER-2) overexpression. After curative surgery, $42 \%$ of patients (19/45) received adjuvant chemotherapy; $77 \%(27 / 35)$ received hormone therapy. Five out of ten patients with HER-2 overexpressing tumors did not receive adjuvant anti-HER-2 therapy, while two out of four patients with HER-2 overexpressing tumors received palliative trastuzumab for recurrent and metastatic disease. Letrozole was used for one patient in the palliative setting. The median overall survival durations were 7.2 years (range, 0.6 to 17.0 years) in patients with localized disease and 2.9 years (range, 0.6 to 4.3 years) in those with recurrent or metastatic disease.

\section{Conclusion}

Anti-HER-2 and hormonal therapy, except tamoxifen, have been underutilized in Korean MBC patients compared to female breast cancer patients. With the development of precision medicine, active treatment with targeted agents should be applied. Further investigation of the unique pathobiology of MBC is clinically warranted.

\section{Key words}

Correspondence: In Sook Woo, MD, PhD Department of Internal Medicine, Yeouido St. Mary's Hospital, College of Medicine, The Catholic University of Korea, 10 63-ro, Yeongdeungpo-gu, Seoul 07345, Korea Tel: 82-2-3779-1574

Fax: 82-2-780-3132

E-mail: insookwoo@catholic.ac.kr

Received October 26, 2015

Accepted March 22, 2016

Published Online April 11, 2016 


\section{Introduction}

Since the early 2000s, the incidence of female breast cancer (FBC) in the United States has been decreasing, a phenomenon attributed to a decline in the use of hormone replacement therapy among postmenopausal women [1]. In contrast, according to the Surveillance, Epidemiology and End Result and the United Kingdom Association of Cancer Registries databases, between the 1970s and 2000s, the incidence of male breast cancer (MBC) increased from $0.1 \%$ to $1 \%[2,3]$.
In Korea, according to the annual report of cancer statistics, the incidence of MBC increased from $0.041 \%$ in 1999 to $0.068 \%$ in 2012 [4]. Furthermore, studies indicate that the incidence of $\mathrm{MBC}$ in Korea was as high as $0.27 \%-0.58 \%$ [5-7].

Because $\mathrm{MBC}$ is rare, the general treatment approaches for MBC are based upon those for FBC [8]. Since the development of human epidermal growth factor receptor 2 (HER-2)targeted agents such as trastuzumab, pertuzumab, trastuzumab-DM1, and lapatinib, and hormone agents such as aromatase inhibitors, the treatment outcomes for FBC have

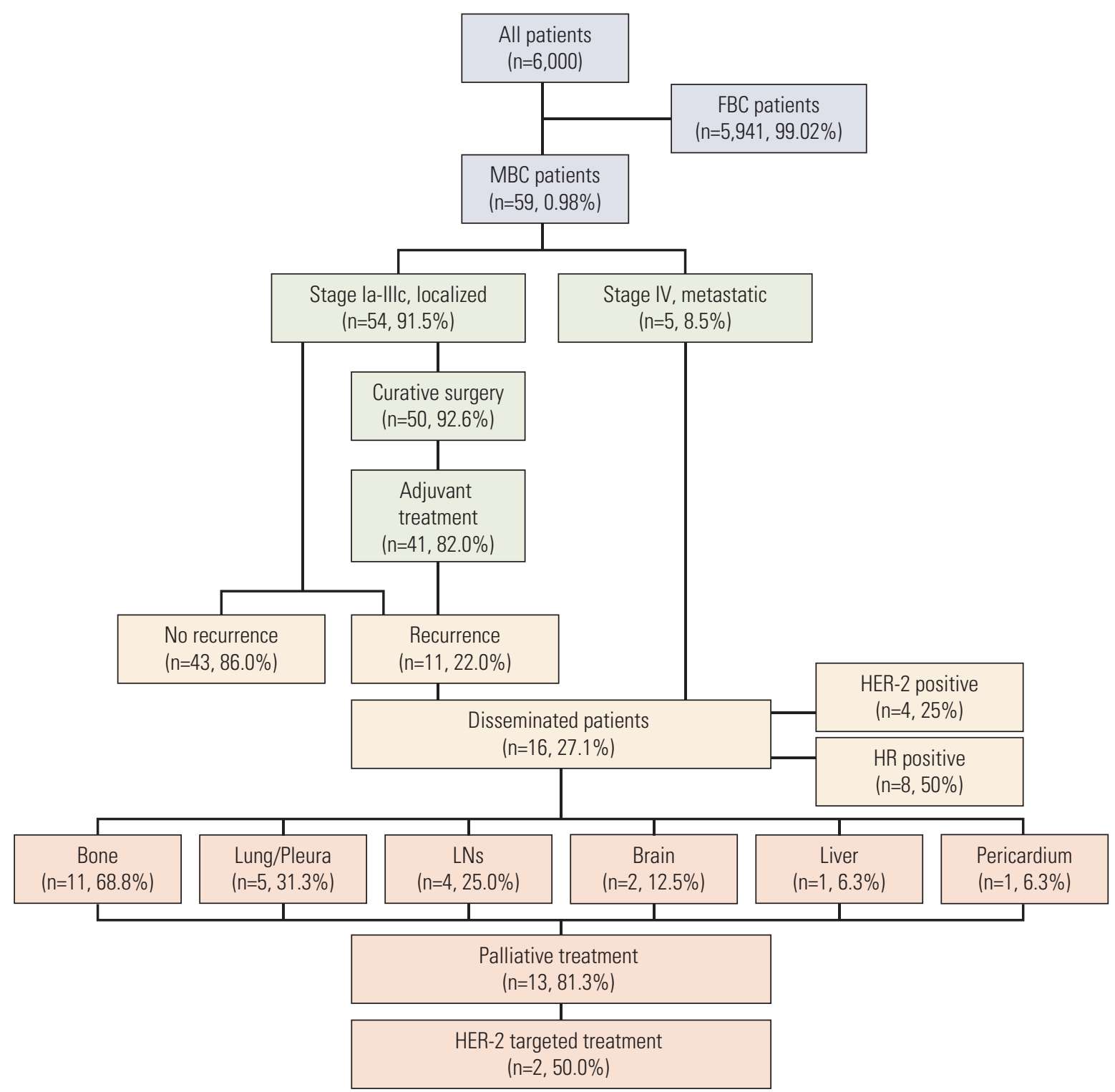

Fig. 1. Patients diagram. FBC, female breast cancer; $M B C$, male breast cancer; HER-2, human epidermal growth factor receptor 2; HR, hormone receptor; $\mathrm{LN}$, lymph node. 
improved remarkably. However, these treatments have not been fully implemented in MBC patients because of the lack of clinical trials investigating $\mathrm{MBC}$, as well as concerns that the tumor biology may be different between the sexes. Moreover, studies have reported contradictory treatment outcomes between MBC and FBC patients [9-11].

In the present study, we analyzed data from seven Korean institutions, spanning a 20-year period, to evaluate the clinicopathologic characteristics, treatment strategies, and clinical outcomes of MBC patients in the modern treatment era. The treatment outcomes and prognostic factors affecting survival were also evaluated.

\section{Materials and Methods}

\section{Patients}

Among 6,000 patients with breast cancer treated between 1995 and 2014 at Seoul St. Mary's Hospital (Seoul, Korea), Yeouido St. Mary's Hospital (Seoul, Korea), Incheon St. Mary's Hospital (Incheon, Korea), Uijeongbu St. Mary's Hospital (Uijeongbu, Korea), Bucheon St. Mary's Hospital (Bucheon, Korea), St. Vincent's Hospital (Suwon, Korea), and Daejeon St. Mary's Hospital (Daejeon, Korea), data for 59 MBC patients were collected for analysis (Fig. 1).

The patients' medical records were reviewed in July 2015 to obtain the age at diagnosis, complaints at the time of diagnosis, symptom duration, family history of cancer, marital status, smoking habits, alcohol intake, pathologic features, treatments, and clinical course of the disease. Approval for this study was obtained from the Institutional Review Board of The Catholic Medical Center (No. XC15RIMI0046O). The informed consent was waived because of its retrospective design of the study.

\section{Statistical analyses}

All statistical analyses were performed using SPSS ver. 19.0 (IBM Co., Armonk, NY). Descriptive statistics are presented for the baseline characteristics. To compare two groups, we used the chi-square test for categorical variables, the $t$ test for continuous variables, and the non-parametric Mann-Whitney $U$ test when the assumption of normality was questionable. Overall survival (OS) was defined as the time interval from the date of diagnosis to that of death from any cause or the last follow-up date. Disease-free survival (DFS) was defined as the time interval between the date of diagnosis and that of the first recurrence or death from any cause. Survival data were estimated using the Kaplan-Meier method.
Cox regression analysis was used to identify factors associated with the risk of death. All p-values presented are twosided, and p-values of $<0.05$ were considered statistically significant.

\section{Results}

\section{Patient characteristics}

The baseline patient characteristics are summarized in Table 1 . The median symptom duration was 5 months (range, 1 to 36 months). Left-sided tumors accounted for 53\% of cases. The most common complaint at diagnosis was a palpable breast mass. The majority of patients had stage II, clinical (c) T1, and cN0 disease at the time of diagnosis. In the six patients who had a family history of breast cancer, the age at onset of the disease tended to be earlier than in those who did not have a family history of the disease ( 52.3 years vs. 65.4 years, $\mathrm{p}=0.051$ ). There were no differences in the tumor grade, hormone receptor (HR) positivity, or HER-2 overexpression status between patients with or without a family history of breast cancer. Among patients with initial stage IV disease, four demonstrated bone metastases and one demonstrated apparent liver metastasis.

\section{Histopathology}

The histopathological characteristics are in Table 2. The most common MBC histological subtype was infiltrating ductal carcinoma (74.5\%). Most tumors showed poor or moderate differentiation $(35.6 \% / 37.3 \%)$. The pathologic (p) T classification data was available for 51 tumors, and most of them were $\mathrm{pT} 1$ or $\mathrm{pT} 2$. The median tumor diameter was 2.0 $\mathrm{cm}$ (range, 0.4 to $9.0 \mathrm{~cm}$ ). The median number of lymph nodes removed during surgery was 10 (range, 0 to 35). Nineteen patients had pathologically positive lymph nodes, and the mean number of involved lymph nodes was 1.3 (standard deviation, 2.6; range, 0 to 11). Eighteen of 59 patients had histologic evidence of lymphatic invasion.

Among the 42 patients with available data, $90.5 \%$ were positive for estrogen receptor (ER) and $64.3 \%$ for progesterone receptor (PR) expression. All PR-positive patients were ER-positive. Of the 40 patients with HER-2 expression data obtained using immunohistochemical or fluorescent in situ hybridization analyses, $27.5 \%$ showed HER-2 overexpression. There were no differences in the age distribution, family history of breast cancer, alcohol intake, smoking, and disease stage according to the HR positivity and HER-2 overexpression statuses. 
Table 1. Patient characteristics

\section{Characteristic}

No. $(\%)(\mathrm{n}=59)$

\begin{tabular}{|cc|}
$\begin{array}{l}\text { Age at diagnosis, median (range, yr) } \\
\text { Diagnosis period }\end{array}$ & $66(24-87)$ \\
\hline $1995-1999$ & $8(13.6)$ \\
$2000-2004$ & $13(22.0)$ \\
\hline $2005-2010$ & $17(28.8)$ \\
\hline $2010-2014$ & $21(35.6)$ \\
\hline Laterality (n=57) & \\
\hline Right & $25(43.9)$ \\
\hline Left & $31(54.4)$ \\
\hline Both & $1(1.7)$ \\
Marital status & \\
\hline Never married & $2(3.4)$ \\
\hline Married & $33(55.9)$ \\
\hline NA & $24(40.7)$ \\
\hline
\end{tabular}

Alcohol

Current alcohol user $16(27.1)$

No alcohol 18 (30.5)

NA $25(42.4)$

Tobacco

Current smoker $10(16.9)$

Ex-smoker $10(16.9)$

Never smoker $29(49.2)$

NA

$10(16.9)$

Chief complaint at the time of diagnosis

Palpable mass

$43(72.9)$

Nipple discharge $3(5.1)$

Nipple tenderness $\quad 1(1.7)$

Back pain $1(1.7)$

Dyspnea $1(1.7)$

NA $10(16.9)$

Duration of symptoms, median (range, mo) 5 (1-36)

Less than $1 \mathrm{mo} \quad 13(22.0)$

$1-6 \mathrm{mo} \quad 13(22.0)$

6 mo-1 yr 8 (13.6)

$1-2 \mathrm{yr} \quad 2(3.4)$

$2-3$ yr $4(6.8)$

Over 3 yr $3(5.1)$

NA $16(27.1)$

Family history of cancer

No family history $33(55.9)$

Family history of cancer $\quad 6(10.2)$

NA 20 (33.9)

Clinical stage

I $17(28.8)$

II $26(44.1)$

III $6(10.2)$

IV $5(8.5)$

NA $5(8.5)$
Table 1. Continued

\begin{tabular}{lc} 
Characteristic & No. $(\%)(\mathbf{n}=59)$ \\
Clinical T stage & \\
T1 & $28(47.5)$ \\
T2 & $20(33.9)$ \\
T3 & $4(6.8)$ \\
NA & $7(11.9)$ \\
Clinical N stage & \\
N0 & $27(45.8)$ \\
N1 & $19(32.2)$ \\
N2 & $2(3.4)$ \\
N3 & $4(6.8)$ \\
NA & $7(11.9)$ \\
\hline
\end{tabular}

NA, not available.

\section{Surgery and adjuvant treatment}

Details of the treatments administered are in Table 3. Fifty patients underwent curative surgery, including modified radical mastectomy or total mastectomy. Adjuvant chemotherapy was indicated for node-positive tumors or tumors $>1 \mathrm{~cm}$ in size, and 19 of $45(42.2 \%)$ such patients received adjuvant chemotherapy. Of these 19 patients, 15 received anthracycline-based regimens, as follows: seven received adriamycin and cyclophosphamide followed by docetaxel; five received fluorouracil, adriamycin, and cyclophosphamide; one received epirubicin and docetaxel; one received epirubicin and carboplatin; and one underwent adriamycin monotherapy. Two patients were administered a combination of cyclophosphamide, methotrexate, and fluorouracil. One patient received paclitaxel monotherapy and another received oral fluorouracil monotherapy. There were no differences in the age distribution, family history of breast cancer, alcohol intake, smoking, and stage between patients treated with and without adjuvant chemotherapy. Node-positive disease was observed more frequently in patients treated with adjuvant chemotherapy $(\mathrm{p}=0.007)$.

Among 35 patients with HR-positive tumors after curative surgery, $27(77.1 \%)$ received adjuvant hormone therapy. One patient did not undergo any adjuvant treatment because of poor compliance. Eleven patients recieved both adjuvant chemotherapy and hormone treatment. In seven patients, the reason for not administering adjuvant hormone treatment was not identified owing to insufficient medical records. There were no differences in the age distribution, family history of breast cancer, alcohol intake, and stage between patients treated with and without adjuvant hormone treatment. However, alcohol consumption was observed more frequently in patients treated with adjuvant hormone therapy $(p=0.019)$. Among 10 patients with HER-2 overexpress- 
Table 2. Histopathologic characteristics

\section{Characteristic}

No. $(\%)(\mathrm{n}=59)$

\section{Histology}

Infiltrating ductal carcinoma

$44(74.5)$

Papillary carcinoma

Ductal carcinoma in situ

$4(6.8)$

Mucinous carcinoma

$2(3.4)$

Cribriform carcinoma

$2(3.4)$

Sarcoma

$1(1.7)$

Carcinoma, NOS

$2(3.4)$

Grade

$\begin{array}{lc}\text { Well } & 5(8.5) \\ \text { Moderately } & 22(37.3) \\ \text { Poorly } & 21(35.6) \\ \text { NA } & 11(18.7)\end{array}$

TNM stage

$$
\text { I }
$$

$17(28.8)$

$$
\text { II }
$$

III

$26(44.1)$

$6(10.2)$

$5(8.5)$

NA

$5(8.5)$

Pathologic T stage $(n=50)$

T1

T2

$27(54.0)$

$18(36.0)$

T3

$1(2.0)$

NA

$25(50.0)$

$19(38.0)$

$2(4.0)$

$3(6.0)$

$1(2.0)$

NA

$18(54.5)$

15 (45.5)

Patic invasion $(n=33)$

Positive

Negative

$38(90.5)$

Negative

Positive

15 (35.7)

Negative

$15(35.7)$

Positive

HER-2 overexpression $(n=40)$

Negative

$29(72.5)$

Positive

$11(27.5)$

NOS, not otherwise specified; NA, not available; HER-2, human epidermal growth factor receptor 2 .
Table 3. Treatment and survival status

\begin{tabular}{lc} 
Characteristic & No. $(\%)(\mathbf{n}=59)$ \\
Surgery $(\mathbf{n}=50)$ & \\
Sentinel lymph node biopsy & $5(10.0)$ \\
Modified radical mastectomy & $43(86.0)$ \\
Total mastectomy & $5(10.0)$ \\
Lumpectomy & $2(4.0)$ \\
Adjuvant treatment (n=41) & \\
Chemotherapy & $8(19.5)$ \\
Hormone therapy & $21(51.2)$ \\
Radiotherapy & $1(2.4)$ \\
Both chemotherapy and hormone therapy & $11(26.8)$ \\
Status at last follow-up & \\
No evidence of disease & $43(72.9)$ \\
Alive with disease & $5(8.5)$ \\
Dead of disease & $8(13.6)$ \\
Dead of other causes & $3(5.1)$ \\
\hline
\end{tabular}

ing tumors, five were indicated for adjuvant trastuzumab; however, they did not receive trastuzumab.

\section{Recurrence and palliative treatment}

Eleven patients $(22.0 \%)$ with localized disease who underwent curative surgery had recurrence at the time of analysis. The most frequent recurrence site was the bone (7 patients, $63.6 \%)$, followed by the lungs / pleura $(5,45.5 \%)$ and brain $(2$, $18.2 \%$ ). Local relapse involving the lymph nodes occurred in three patients $(27.3 \%)$. HR-positive tumors occurred in $54.5 \%$ $(6 / 11)$ and $18.2 \%(2 / 11)$ of patients with recurrence had HER-2 overexpressing tumors. Between patients who experienced recurrence and those who did not, there were no differences in the HR positivity and HER-2 overexpression status.

In patients with initial stage IV disease, the most frequent metastatic site was the bone (4 patients), followed by the lymph node, liver, and pericardium (1 patient each). In the 16 patients with recurrent or metastatic disease, $13(81.3 \%)$ received palliative treatment. Two patients did not receive anti-cancer treatment because of underlying pneumonia and poor performance status. One patient refused treatment and received best supportive care. In the four patients diagnosed with HER-2 overexpressing disseminated tumors, two received HER-2 targeted palliative treatment. Among eight patients with HR-positive tumors, one patient, who experienced multiple bone metastases under adjuvant chemotherapy and tamoxifen, received palliative letrozole alone without gonadotropin-releasing hormone $(\mathrm{GnRH})$ agonists or orchiectomy, surviving 21 months progression-free. Five patients received palliative chemotherapy after progression 
A
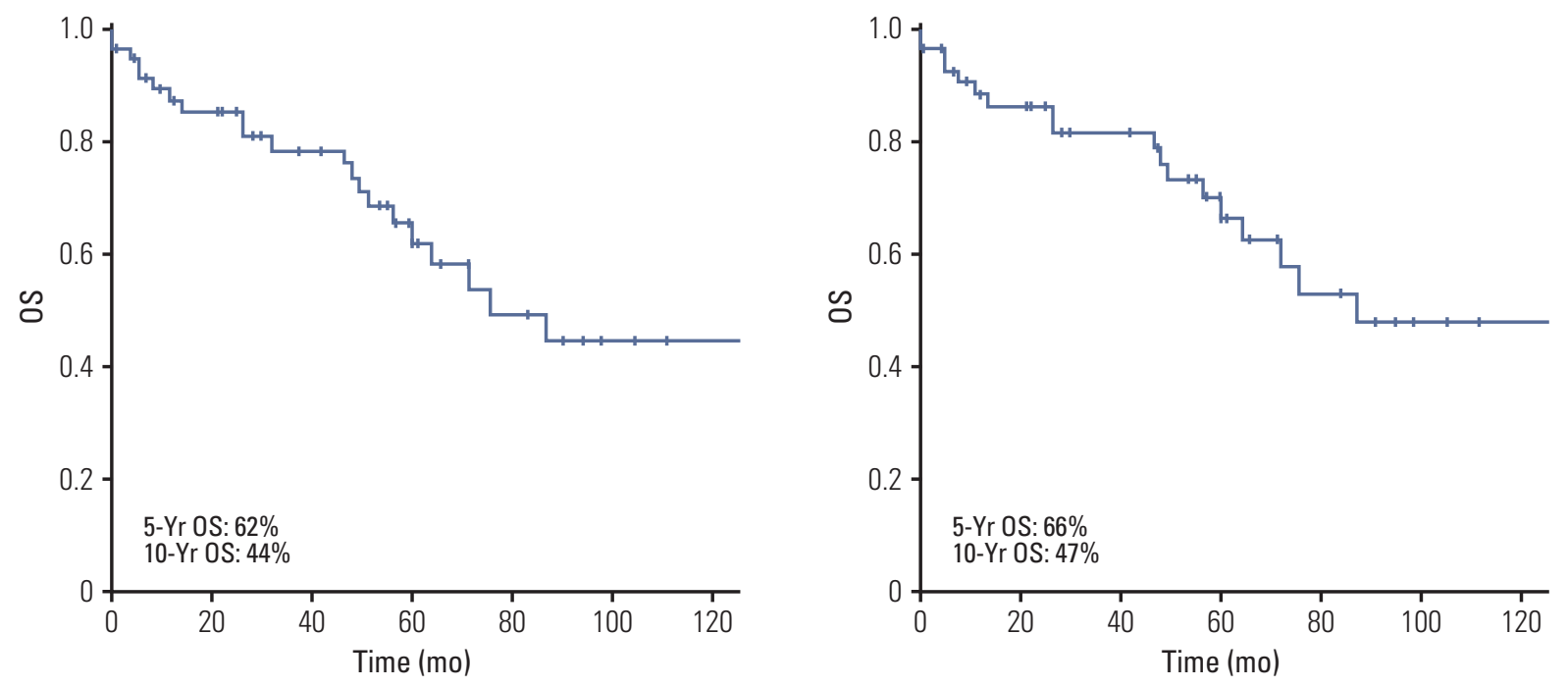

C

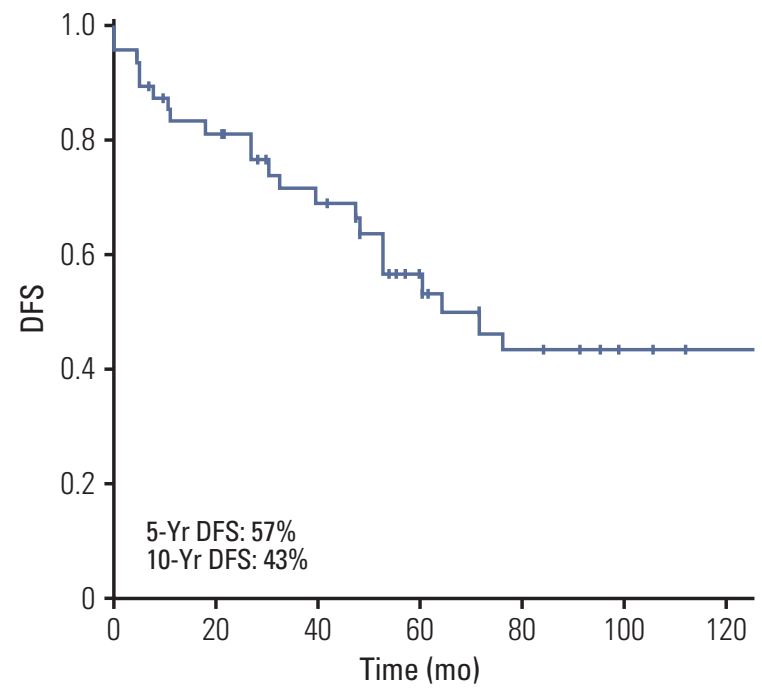

Fig. 2. (A) Overall survival (OS) of total male patients with breast cancer. (B) OS of male patients with localized breast cancer. (C) Disease-free survival (DFS) of male patients with localized breast cancer.

under hormone therapy. One patient did not receive any treatment.

\section{Survival outcomes}

Eleven patients (18.7\%) had died at the time of analysis. The median OS of the 59 patients was 6.3 years (range, 0.6 to 17.0 years). The 5- and 10 -year OS rates were $62 \%$ and $44 \%$ (Fig. 2A). The median OS and DFS in patients with localized disease were 7.2 years (range, 0.6 to 17.0 years) and 2.9 years (range, 0.6 to 4.3 years). The 5- and 10-year OS rates for patients with localized disease were $66 \%$ and $47 \%$ (Fig. 2B). The 5- and 10-year DFS rates for patients with localized disease were $57 \%$ and $43 \%$ (Fig. 2C). The median OS in patients with metastatic disease was 2.9 years (range, 0.6 to 4.3 years), with $50 \%$ of the 3-year OS rates.

A family history of breast cancer, alcohol intake, smoking, age, and axillary lymph node involvement did not affect the OS or DFS. There was no difference in OS between patients treated with and without adjuvant chemotherapy among the 
Table 4. Cox regression analysis of the factors associated with overall survival in male breast cancer $(n=59)$

\begin{tabular}{lccc} 
Characteristic & Crude HR & 95\% CI & p-value \\
Stage (I vs. II, III, IV) & 6.11 & $1.151-32.428$ & $0.034^{*}$ \\
Age (<65 yr vs. $\geq 65$ yr) & 0.64 & $0.216-1.951$ & 0.442 \\
Smoking state (never vs. ever) & 1.66 & $0.522-5.308$ & 0.389 \\
LN involvement (absent vs. present) & 1.33 & $0.633-1.338$ & 0.633 \\
Lymphatic invasion (absent vs. present) & 4.42 & $0.514-37.966$ & 0.176 \\
ER status (positive vs. negative) & 27.04 & $0.001-959,175.267$ & 0.537 \\
\hline HER2 status (negative vs. positive) & 0.39 & $0.741-2.152$ & 0.280 \\
Axillary lymph node dissection (done vs. not done) & 1.34 & $0.405-4.418$ & 0.633 \\
\hline Adjuvant treatment (done vs. not done) & 1.81 & $0.555-5.878$ & 0.327 \\
\hline
\end{tabular}

HR, hazard ratio; CI, confidence interval; LN, lymph node; ER, estrogen receptor; HER-2, human epidermal growth factor receptor 2. * $\mathrm{p}<0.05$.

indicated patients ( $\mathrm{p}=0.081$ and $\mathrm{p}=0.218$, respectively).

In patients with positive HR status, there was no significant difference in DFS between patients who received adjuvant hormone therapy and those who did not $(\mathrm{p}=0.636)$. However, in terms of OS, adjuvant hormone therapy improved survival in patients with positive HR status (8.5 years; $95 \%$ confidence interval [CI], 4.49 to 12.58 vs. 4.2 years; 95\% CI, 3.09 to 5.29; $\mathrm{p}=0.047$ ).

\section{Factors associated with OS}

The Cox regression analysis of the factors associated with OS is shown in Table 4. Stage was the only factor significantly associated with survival $(p=0.034)$ in the univariate analysis. Patients with early stage and locally advanced breast cancer showed better OS than those with metastatic disease (9.9 years; 95\% CI, 7.97 to 11.97 vs. 2.8 years; $95 \% \mathrm{CI}$, 1.27 to $4.51 ; \mathrm{p}=0.019)$. The HR and HER-2 overexpression status had no effect on the OS $(p=0.087$ and $p=0.280$, respectively).

\section{Discussion}

In the present study, over a 20 -year period, $\mathrm{MBC}$ patients were detected in $0.98 \%$ of total breast cancer patients across seven institutions in Korea. The global incidence rates of MBC vary widely by location and ethnicity. In Japan, the rate is reported at $0.49 \%$; in the United States and Europe, it is $1 \%$; and across African nations, it is reported at 5\%-15\% $[2,3,9,12]$. As the global population ages and diagnostic techniques improve, the global incidence rate of MBC continues to rise.

The distinct histologic characteristics and related biology of MBC compared with FBC have not been fully described, and, therefore, knowledge is still lacking regarding $\mathrm{MBC}$.

The median age of MBC onset in our study, 66 years, was consistent with the literature (62-69 years), and is much later than that reported for FBC ( $<50$ years) [10,12]. This late onset might be due to a lack of awareness of the early signs of MBC, which may lead to delayed screening and diagnosis [10]. The median symptom duration was reported as 18.6 months by Baek et al. [5] and 13 months by Cho et al. [6], longer than the median duration of 5 months in the present study. This prolonged period of symptom duration likely contributes to the differences in stage distribution between MBC and FBC patients and may be the reason why metastatic disease is more frequently detected at the time of diagnosis in MBC patients. The rate of disseminated disease in the present study $(8.5 \%)$ was similar to that reported by Nahleh et al. [13] (8.9\%) and Miao et al. [10] (10.9\%), whereas the rate for FBC patients was lower (5\%-6\%).

The rate of familial breast cancer in $\mathrm{MBC}$ is reported as $15 \%-20 \%$, higher than for FBC $(7 \%)[8,14]$. In the present study, MBC patients with a family history of breast cancer $(8.5 \%)$ had earlier disease onset compared to MBC patients without a family history, although the difference was not significant. Other clinicopathologic features, as well as survival, were not different by family history. These findings were consistent with those of Hill et al. [14], who did not find any significant effect of family history on stage at diagnosis or survival outcome in MBC patients. Meanwhile, according to Ottini et al. [15], MBC patients with BRCA2 mutations were more likely to have a family history of breast or ovarian cancers, higher tumor grades, and HER-2 overexpression when compared to those with BRCA1 mutations [15]. Further studies evaluating the $B R C A 1 / 2$ mutation status in MBC are clinically warranted to understand the genotype-phenotype correlations in $\mathrm{MBC}$ patients.

HER-2 overexpression occurs in approximately $25 \%$ of 
FBC cases, and there are contradictory reports about the rate of HER-2 overexpression in MBC. Some researchers suggest that the HER-2 overexpression rates in MBC patients may be higher (30\%-56\%) than thoses in FBC patients [16], while others report lower HER-2 overexpression rates in MBC patients $(2 \%-18 \%)[8,17]$. According to Park et al. [18], among 10 Korean MBC patients, five (50\%) demonstrated HER-2 overexpression. Furthermore, Cho et al. [6] showed that four of 14 MBC patients $(28.5 \%)$ had HER-2 overexpression. In the present study, 11 of $40 \mathrm{MBC}$ patients (27.5\%) overexpressed HER-2. Discrepancies in the HER-2 overexpression rates between these studies are likely explained by the small study populations of each study as well as heterogeneity in the included stages and ethnicities and epidemiological differences.

Since a regression of metastasis after orchiectomy was firstly reported in 1942, orchiectomy has become the standard treatment for advanced disease in $\mathrm{MBC}$ and results in a 55\% response rate [19]. Following reports of substantial response with anti-estrogen, hormonal therapy is an appealing alternative to surgery, avoiding psychological, morbidity, and mortality risks associated with surgery [20]. Tamoxifen is the standard treatment for adjuvant hormonal therapy over aromatase inhibitor in MBC as well as for metastatic disease. The efficacy of aromatase inhibitors in $\mathrm{MBC}$ is ambivalent because of differences in estrogen repression between the sexes. Administration of anastrozole in healthy young men reduced estradiol concentrations by only $50 \%$, limiting the anti-tumor effect of anastrozole, whereas in women, it completely suppressed estrogen levels [21]. In a study of Zagouri et al. [22] with 23 metastatic MBC patients, no significant effects of combination of GnRH analogue and aromatase inhibitor were noted on OS and progression-free survival (PFS). In contrary, in a larger study of 60 metastatic MBC patients, patients treated with aromatase inhibitor in combination with GnRH analogues showed better PFS and OS compared with aromatase inhibitor alone [23]. Currently, the National Comprehensive Cancer Network guidelines suggest that in men with advanced breast cancer, the use of aromatase inhibitors is ineffective without concomitant suppression of testicular steroidogenesis [24]. Interestingly, in our study, one patient with recurrence of multiple bone metastases was on letrozole for 21 months and survived for 90 months after recurrence.

Some studies suggest that $\mathrm{MBC}$ has a worse outcome than FBC [11]. The presumption is that the small volume of breast tissue in men, which would permit cancer cells easy access to the lymphatic system and direct extension to the muscles and chest wall, leads to high recurrence rates of cancer [11]. Another explanation could be that $\mathrm{MBC}$ is initially diagnosed at a much later age, and that these patients are more likely to have a higher risk of cardiovascular and pulmonary comorbidities $[8,9]$. Similarly, because of the advanced age, the use of radiotherapy is more limited in MBC patients compared with in FBC patients [8,9]. Delayed diagnosis and treatment, and a lack of knowledge of the unique characteristics of MBC could result in a less favorable prognosis for MBC patients compared with FBC patients. Estimates for 5-year survival for MBC patients are reported at 40\%-65\%, which was similar to $62 \%$ of our present study [9]. However, some studies evaluating age- and stage-matched patients did not find any significant survival differences between MBC and FBC patients $[9,10]$.

The strengths of the present study are the large sample size and the long-term survival data for MBC patients compared to previous reports in Korea. The Korean male population is well represented because the data were collected from seven institutions, including two tertiary referral and five community hospitals, which provide medical services to mixed urban and rural populations.

However, the study has limitations, including the lack of information regarding molecular characteristics such as $B R C A 1 / B R C A 2$ mutations and the p53 status. In addition, the pathologic data were collected in a retrospective manner and could not be reviewed for each patient.

At present, the systemic treatment guideline recommended for $\mathrm{MBC}$ is almost the same as that for postmenopausal FBC. However, according to this study, other hormonal agents, except tamoxifen and anti-HER-2 therapy, have been underutilized in MBC patients compared with FBC. The reason may be that many of the therapeutic agents used for breast cancer have been specified for FBC, as there is little clinical trial evidence for MBC and therefore limitation of national insurance guidelines. It is logistically difficult to perform prospective randomized clinical trials for the development of the therapeutic regimens for MBC because of paucity of cases. Therefore, the physician and patient should be aware of these limitations and follow a more active approach for treating this disease.

\section{Conclusion}

In conclusion, in this study, the HR-positive rate was higher in patients with $\mathrm{MBC}$ compared to that reported in patients with $\mathrm{FBC}$, whereas the HER-2 overexpression rate was similar. The survival outcomes in MBC patients were similar to those reported in FBC patients. Personalized treatment should be performed for $\mathrm{MBC}$ in concordance with the advances in the treatment of FBC. 


\section{Conflicts of Interest}

Conflict of interest relevant to this article was not reported.

\section{Author Details}

${ }^{1}$ Department of Internal Medicine, Incheon St. Mary's Hospital, Incheon, ${ }^{2}$ Department of Internal Medicine, Seoul St. Mary's Hospital, Seoul, ${ }^{3}$ Department of Internal Medicine,
Yeouido St. Mary's Hospital, Seoul, ${ }^{4}$ Department of Internal Medicine, Uijeongbu St. Mary's Hospital, Uijeongbu, ${ }^{5}$ Department of Internal Medicine, St. Vincent's Hospital, Suwon, ${ }^{6}$ Department of Internal Medicine, Bucheon St. Mary's Hospital, Bucheon, ${ }^{7}$ Department of Internal Medicine, Daejeon St. Mary's Hospital, Daejeon, ${ }^{8}$ Department of Surgery, St. Vincent's Hospital, Suwon, ${ }^{9}$ Department of Surgery, Uijeongbu St. Mary's Hospital, Uijeongbu, ${ }^{10}$ Department of Surgery, Yeouido St. Mary's Hospital, Seoul, ${ }^{11}$ Department of Surgery, Seoul St. Mary's Hospital, Seoul, ${ }^{12}$ Department of Surgery, Bucheon St. Mary's Hospital, Bucheon, ${ }^{13}$ Department of Radiation Oncology, Yeouido St. Mary's Hospital, Seoul, Korea

\section{References}

1. Ravdin PM, Cronin KA, Howlader N, Berg CD, Chlebowski $\mathrm{RT}$, Feuer EJ, et al. The decrease in breast-cancer incidence in 2003 in the United States. N Engl J Med. 2007;356:1670-4.

2. Stang A, Thomssen C. Decline in breast cancer incidence in the United States: what about male breast cancer? Breast Cancer Res Treat. 2008;112:595-6.

3. Speirs V, Shaaban AM. The rising incidence of male breast cancer. Breast Cancer Res Treat. 2009;115:429-30.

4. Ministry of Health and Welfare; Korea Central Cancer Registry; National Cancer Center. Annual report of cancer statistics in Korea in 2012. Goyang: National Cancer Center; 2012.

5. Baek JM, Sung GY, Lee DS, Kim W, Park IY, Jung SS, et al. Male breast cancer. J Breast Cancer. 2005;8:123-7.

6. Cho J, Han W, Ko E, Lee JW, Jung SY, Kim EK, et al. The clinical and histopathological characteristics of male breast cancer patient. J Breast Cancer. 2007;10:211-6.

7. Lee PC, Son BH, Kwon JN, Lee WB, Lee KM. Clinical features of the male breast cancer- 13 cases. J Korean Breast Cancer Soc. 2004;7:193-8.

8. Gomez-Raposo C, Zambrana Tevar F, Sereno Moyano M, Lopez Gomez M, Casado E. Male breast cancer. Cancer Treat Rev. 2010;36:451-7.

9. Fentiman IS, Fourquet A, Hortobagyi GN. Male breast cancer. Lancet. 2006;367:595-604.

10. Miao H, Verkooijen HM, Chia KS, Bouchardy C, Pukkala E, Laronningen $S$, et al. Incidence and outcome of male breast cancer: an international population-based study. J Clin Oncol. 2011;29:4381-6.

11. Scott-Conner CE, Jochimsen PR, Menck HR, Winchester DJ. An analysis of male and female breast cancer treatment and survival among demographically identical pairs of patients. Surgery. 1999;126:775-80.

12. Ioka A, Tsukuma H, Ajiki W, Oshima A. Survival of male breast cancer patients: a population-based study in Osaka, Japan. Jpn J Clin Oncol. 2006;36:699-703.

13. Nahleh ZA, Srikantiah R, Safa M, Jazieh AR, Muhleman A,
Komrokji R. Male breast cancer in the veterans affairs population: a comparative analysis. Cancer. 2007;109:1471-7.

14. Hill A, Yagmur Y, Tran KN, Bolton JS, Robson M, Borgen PI. Localized male breast carcinoma and family history: an analysis of 142 patients. Cancer. 1999;86:821-5.

15. Ottini L, Silvestri V, Rizzolo P, Falchetti M, Zanna I, Saieva C, et al. Clinical and pathologic characteristics of BRCA-positive and BRCA-negative male breast cancer patients: results from a collaborative multicenter study in Italy. Breast Cancer Res Treat. 2012;134:411-8.

16. Onami S, Ozaki M, Mortimer JE, Pal SK. Male breast cancer: an update in diagnosis, treatment and molecular profiling. Maturitas. 2010;65:308-14.

17. Ottini L, Rizzolo P, Zanna I, Falchetti M, Masala G, Ceccarelli $\mathrm{K}$, et al. BRCA1/BRCA2 mutation status and clinical-pathologic features of 108 male breast cancer cases from Tuscany: a population-based study in central Italy. Breast Cancer Res Treat. 2009;116:577-86.

18. Park HL, Kwak JY, Lee SD, Nam SJ, Yang JH. Male breast cancer-10 cases. J Korean Breast Cancer Soc. 2003;6:303-7.

19. Jaiyesimi IA, Buzdar AU, Sahin AA, Ross MA. Carcinoma of the male breast. Ann Intern Med. 1992;117:771-7.

20. Giordano SH, Valero V, Buzdar AU, Hortobagyi GN. Efficacy of anastrozole in male breast cancer. Am J Clin Oncol. 2002;25:235-7.

21. Mauras N, O'Brien KO, Klein KO, Hayes V. Estrogen suppression in males: metabolic effects. J Clin Endocrinol Metab. 2000;85:2370-7.

22. Zagouri F, Sergentanis TN, Koutoulidis V, Sparber C, Steger GG, Dubsky P, et al. Aromatase inhibitors with or without gonadotropin-releasing hormone analogue in metastatic male breast cancer: a case series. Br J Cancer. 2013;108:2259-63.

23. Di Lauro L, Pizzuti L, Barba M, Sergi D, Sperduti I, Mottolese $\mathrm{M}$, et al. Role of gonadotropin-releasing hormone analogues in metastatic male breast cancer: results from a pooled analysis. J Hematol Oncol. 2015;8:53. 
24. National Comprehensive Cancer Network. NCCN Clinical Practice Guidelines in Oncology Breast Cancer [Internet]. Fort Washington, PA: National Comprehensive Cancer Network; c2015 [cited 2016 Dec 30]. Available from: http://www.nccn .org/professionals/ physician_gls?PDF?brastt.pdf. 